\title{
Energy Losses in Superconductors
}

\author{
Charles Dean, Milind N. Kunchur \\ Department of Physics and Astronomy, University of South Carolina, Columbia, USA \\ Email: kunchur@sc.edu
}

Received 22 March 2016; accepted 22 May 2016; published 25 May 2016

Copyright (C) 2016 by authors and Scientific Research Publishing Inc.

This work is licensed under the Creative Commons Attribution International License (CC BY).

http://creativecommons.org/licenses/by/4.0/

(c) (i) Open Access

\begin{abstract}
Absence of macroscopic resistance is the most essential trait of superconductors for their practical applications. Yet several mechanisms lead to dissipation in the superconducting state such as weak links, fluctuations, and the motion of flux vortices in the mixed state of type II materials. Thus it is of practical as well as fundamental interest to explore these mechanisms to the fullest extent and to learn how to control them. Regarding the control of flux motion, most research efforts aim at preventing flux motion by introducing a variety of pinning strategies. In the present work, we discuss the circumstances under which it is possible to exclude vortices in the first place, or to short their motion through intrinsic multi-band effects. Experimental results on two model systems, molybdenum-germanium and magnesium diboride films, are presented to support these strategies.
\end{abstract}

\section{Keywords}

Mixed State, Vortices, Critical Current, Two-Band

\section{Introduction}

The transport of electrical current and levitation are two of the most important areas of practical importance for the application of superconductors. These are based respectively on the two most important fundamental properties of the superconducting state: flux expulsion through the Meissner effect and the vanishing of resistance. Superconductivity for energy applications is mainly concerned with the latter property, as it depends on the ability to carry lossless electrical currents for the purpose of power transmission and for the construction of extremely powerful electromagnets for use in generators and motors, and for other applications such as highenergy particle accelerators and nuclear fusion where intense magnetic fields are required. Superconducting conductors allow a scaling down of size which is of importance for plasma confinement in fusion.

A type-II superconductor enters the mixed state in the presence of a magnetic field $B$ that is higher than the lower critical field $B_{c 1}$. This mixed state consists of quantized flux vortices each containing a quantum of mag- 
netic flux $\Phi_{0}=h / 2 \mathrm{e}$ and with a normal core of radius comparable to the coherence length $\xi$. The motion of the vortices leads to an electric field within the superconductor and hence resistance and power dissipation, rendering the conductor unattractive for lossless current carrying applications. A major effort has been spent in engineering pinning strategies to prevent or greatly reduce the motion of flux vortices. Flux motion also tends to progressively broaden the resistive transition with increasing $B$. We discuss here some intrinsic fundamental phenomena that can alleviate the ill effects of vortex motion.

\section{Exclusion of Vortices and Exotic Vortex States That Reduce Dissipation}

\subsection{Dimensional Enhancement of the Lower Critical Field}

The most straightforward vortex free state arises when the applied $B$ is less than the lower critical field. The origin of $B_{c 1}$ is related to the difference in free energy that results from the insertion of a vortex in the superconductor: the lost condensation energy because of the normal vortex core versus the reduction in the magnetic field energy because less flux is excluded and the $B$ field lines are less crowded. Usually $B_{c 1}$ has a rather low value in extreme type-II superconductors because of the very short coherence length and core size, and consequently smaller volume of missing condensation energy compared to the reduction in magnetic field energy because of typically large demagnetization effects. However this situation can change when a sample with a high aspect ratio such as a film is placed in parallel field. If the film thickness $d$ is much less than the magnetic penetration depth $\lambda$, then the field fully penetrates the cross section and the flux expulsion energy becomes negligible. Under these conditions $B_{c 1}$ becomes greatly enhanced with respect to its usual bulk value and was shown by Abrikosov to be [1]:

its usual bulk value and was shown by Abrikosov to be [1]:

$$
B_{c 1}^{\|}=\left[2 \Phi_{0} / \pi d^{2}\right] \ln (d / \xi)
$$

\subsection{Dimensional Destruction of the Mixed State}

Another vortex excluding condition occurs when the cross section of the sample parallel to the applied B becomes too small. The swirling vortex supercurrents become strangled by the walls. This boosts the current density $j$ at the interface with the walls, which in turn reduces the order parameter $\Delta$. The weakened $\Delta$ leads to a rise in $\xi$ causing the vortex to expand further causing even more current compression at the walls. As a result of this self feeding process, at some point the vortex core explodes and Abrikosov vortices can no longer exist. This process was studied theoretically by Likharev and he found the critical cross-sectional dimension to be [2]:

$$
d_{c}=4.4 \xi(T)
$$

\subsection{High Viscous Coefficient Due to Delocalization of Vortex Electric Fields}

A third novel low-dissipative condition arises in a two-band superconductor when vortex electric fields become delocalized and one of the bands becomes normal but with a high normal conductivity at high $B$. This leads to an anomalous vortex viscosity function that rises linearly with $B$, arising from the additional damping by the quasiparticle sea of the normal band coupled with the unusually long electric field penetration depth $\xi$ that delocalizes the vortex electric fields.

The electric field $E$ generated in the mixed state that leads to resistance, is proportional to the speed of the vortices $v$, so that $E=v B$. This speed in turn is dependent on the driving force per unit vortex length $F_{L}=j_{T} \Phi_{0}$ ( $f o r j_{T}$ perpendicular to $B$ ) exerted by the transport current density $j_{T}$, and on the vortex viscous drag $h v$ so that $j_{T}$ $\Phi_{0}=h v$ in the steady state; here $h$ is the viscous coefficient. A principal cause of the viscous drag is the Ohmic dissipation in and around the vortex core resulting from local electric fields generated by the acceleration of superfluid resulting from the motion of the vortices. In elementary theories for $\eta$, it is assumed that the electric field is confined within a radius comparable to the coherence length $\xi$. As a result the total dissipation for a given $\mathrm{v}$ is simply proportional to the number of vortices and hence $E \alpha B$ and $R \alpha B$. This is the reason flux-flow resistance grows with $\mathrm{B}$ and the resistive transition broadens with $\mathrm{B}$ [3]-[5].

More correctly one should consider the electric field penetration depth $\xi$ to describe the penetration of an electric field from the core of a moving vortex into the surrounding superconducting medium [6] [7]. This $\xi$ is 
controlled by the electron-hole branch-imbalance relaxation time $\tau_{\mathrm{Q}}$, so that $\zeta=\left[D \tau_{Q}\right]^{1 / 2}$, where $D$ is the diffusion constant (the branch imbalance relaxes mainly because of inelastic electron-phonon scattering). A peculiar situation arises when $\zeta \gg \xi$ and especially when $\zeta$ exceeds the vortex spacing $l_{\phi} \approx\left[\Phi_{0} / B\right]^{1 / 2}$. In this case, the electric field will extend throughout the sample and you have scenario of the delocalized vortex electric field [8]. Additionally if the superconductor has two bands, one of which is driven normal and has high normal-state conductivity, then the majority of the dissipation takes place outside the vortex core and throughout the entire volume of the superconductor. This now leads to a viscous coefficient that grows with $B$ and hence the flux-flow resistance does not increase with $B$ and resistive transitions will not broaden.

This scenario is realized in disordered $\mathrm{MgB}_{2}$. Static impurities affect mostly the $\sigma$ band (thereby shortening the coherence length) but have little effect on the weaker $\pi$ band which thus becomes normal with a high conductivity [9]. The $\pi$-band charge carriers thus contribute fully to the dissipation throughout the superconductor volume, whereas the $\sigma$ band dissipation, which is restricted to the region near the vortex core, becomes a smaller contribution. Moreover, $\mathrm{MgB}_{2}$ is expected to have an exceptionally slow inelastic electron-phonon scattering rate because of the unusually high Debye temperature $(\approx 1000 \mathrm{~K})$ which reduces the phonon population at superconducting temperatures (this is also consistent with the almost temperature independent resistivity near $T_{C}$ ). Therefore the electric-field penetration depth is exceptionally large in $\mathrm{MgB}_{2}$, leading to this special scenario where the electric field spills out far beyond the core of vortices and gets "shorted" by the high normal conductivity $\pi$ band. The viscous coefficient thus becomes $\eta \approx \sigma_{\pi} \Phi_{0} B / \pi$ (instead of the $B$-independent Bardeen-Stephen expression $\left.\eta \approx \sigma_{\mathrm{n}} \Phi_{0} B_{c 2} / \pi\right)$ with a flux-flow conductivity $\sigma_{f f}=\eta / B \Phi_{0}$ that is independent of $B$; here $\sigma_{\pi}$ and $\sigma_{\mathrm{n}}$ are the $\pi$-band and conventional single-band normal-state conductivities respectively.

\section{Experimental Techniques}

Transport measurements were conducted in a Cryomech pulsed-tube closed-cycle refrigerator with the magnetic field supplied by a GMW water-cooled electromagnet. The cold tip was positioned within the magnet pole pieces and the magnet was mounted on a rotating platform. All measurements presented here are with $B$ parallel to the film plane directed along the bridge width. The current flows along the bridge length and so is perpendicular to $B$. A video/optical aligner was deviced to ensure that $B / /$ film-plane was within 25 millidegrees. Electrical resistivity measurements were made with a standard dc four-probe method for low currents I or with $0.005 \%$ duty-cycle $20 \mu$ s duration pulses for high I values. The duration of the pulses was chosen to minimize heating while maintaining a high signal-to-noise ratio. Details of the measurement techniques are given in previous review articles [10] [11].

Data are presented on two types of samples: a molybdenum-germanium (MoGe) film and an $\mathrm{MgB}_{2}$ film. The molybdenum-germanium amorphous film of thickness $d=50 \mathrm{~nm}$ was sputtered onto silicon substrates from a $\mathrm{Mo}_{0.79} \mathrm{Ge}_{0.21}$ alloy target [5]. The lithographically patterned bridge had dimensions of length $l=102 \mu \mathrm{m}$ and width $w=6 \mu \mathrm{m}$. This film had a transition temperature $T_{\mathrm{C}}=5.45 \mathrm{~K}$ and normal-state resistance $R_{n}=540 \Omega$. The $\mathrm{MgB}_{2}$ film was grown on $\mathrm{SiC}$ substrates by a hybrid physical-chemical vapour deposition (HPCVD) in-situ process [12]. The films made by this process can have controlled amounts of scattering through carbon doping. The present sample had 22 atomic $\%$ carbon doping. The bridge dimensions for it were $t=170 \mathrm{~nm}, w=15 \mu \mathrm{m}$, and $l=0.8 \mathrm{~mm}$. This film had a $T_{c}=38.7 \mathrm{~K}$ and $R_{n}=36 \Omega$.

\section{Results and Discussion}

We show here one example each for the two sets of special conditions: the vortex-explosion scenario and the two-band mixed state with delocalized electric fields. MoGe is the ideal system to test the former condition. Being an amorphous alloy, it is fully isotropic so that the coherence length that was measured for the perpendicular $\mathrm{B}$ orientation could be taken to be the same for the parallel $\mathrm{B}$ orientation. $\mathrm{MgB}_{2}$ is the ideal system for the second condition because of its long branch-imbalance relaxation time and two-band superconductivity with a weak $\pi$ band that easily turns normal and has a high normal conductivity.

Figure 1(a) shows resistive transition curves [13] for the MoGe film in parallel B field. Notice how the curves converge and plunge around $\mathrm{T}=4.7 \mathrm{~K}$. If one takes the ratio of the nominal thickness of $50 \mathrm{~nm}$ to $\xi(4.7$ $\mathrm{K}$ ), we get $d / \xi=4.2$. This is consistent with Equation 2 for the Likharev vortex explosion condition. Figure 1(b) shows resistive transition curves for the $\mathrm{MgB}_{2}$ film in parallel B field. Notice how the curves shift in an exactly parallel manner without any broadening or change in shape. This is consistent with our observations for the $B$ 


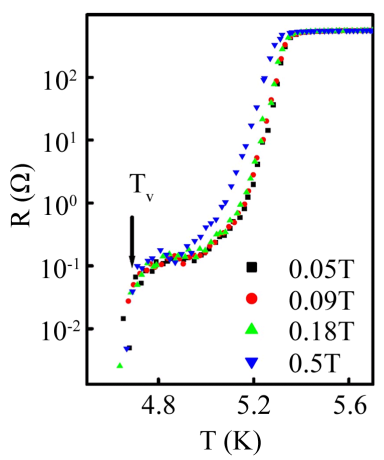

(a)

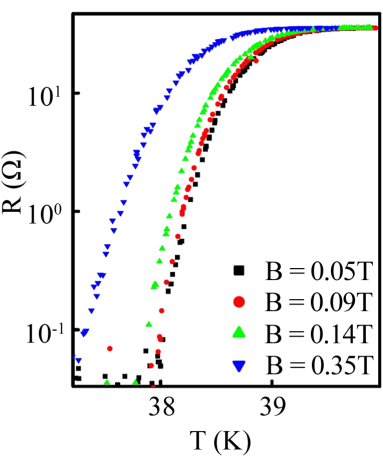

(b)

\begin{abstract}
Figure 1. Contrasting behaviors of a single-band isotropic superconductor (MoGe) in parallel magnetic field and a two-band superconductor with delocalized electric fields $\left(\mathrm{MgB}_{2}\right)$ in parallel magnetic field. (a) MoGe shows a second transition around $\mathrm{T}=4.7 \mathrm{~K}$ (arrow marked $\mathrm{T}_{\mathrm{V}}$ ) where the curves at different $\mathrm{B}$ values converge and plunge. (b) $\mathrm{MgB}_{2}$ shows separate resistive curves that are more more less parallel with no convergence. Details are explained in the text.
\end{abstract}

perpendicular orientation [14].

The effects discussed in here are not well known in the superconductivity community and neither of these effects has been much explored regarding their importance for practical conductors and cables. We hope that this paper brings greater awareness to these newly confirmed fundamental behaviors and that they can be fruitfully exploited for technological purposes.

\title{
5. Conclusion
}

Superconductors are promising materials for power applications such as cables, generators and motors. Reducing their resistance and consequent power dissipation is essential for such applications. A large part of the dissipation arises from flux vortex motion and previous research has been aimed at pinning the vortices to suppress their motion. This paper proposes alternatives to pinning for reducing dissipation through multi-band effects and the exclusion of vortices through core explosion. Experimental measurements are presented that support these strategies.

\section{Acknowledgements}

We gratefully acknowledge J. M. Knight, A. Gurevich, J. Hua, Z. Xiao, A. Pogrebnyakov, P. Orgiani, and X. X. Xi. This work was supported by the US Department of Energy through Grant No. DE-FG02-99ER45763.

\section{References}

[1] Abrikosov, A.A. (1964) On the Lower Critical Field of Thin Layers of Superconductors of the Second Group. Journal of Experimental and Theoretical Physics, 19, 988.

[2] Likharev, K.K. (1979) Superconducting Weak Links. Reviews of Modern Physics, 51, 101. http://dx.doi.org/10.1103/RevModPhys.51.101

[3] Tinkham, M. (1996) Introduction to Superconductivity. McGraw Hill, New York.

[4] Larkin, A.I. and Ovchinnikov, Y.N. (1986) Vortex Motion in Superconductors. In: Langenberg, D.N. and Larkin, A.I., Eds., Nonequilibrium Superconductivity, Chapter 11, Elsevier, Amsterdam.

[5] Liang, M., Kunchur, M.N., Hua, J. and Xiao, Z. (2010) Evaluating Free Flux Flow in Low-Pinning MolybdenumGermanium Superconducting Films. Physical Review B, 82, Article ID: 064502. http://dx.doi.org/10.1103/PhysRevB.82.064502

[6] Hu, C.R. and Thompson, R.S. (1972) Dynamic Structure of Vortices in Superconductors. II. H $\leq$ Hc2. Physical Review $B, 6$, 110. http://dx.doi.org/10.1103/PhysRevB.6.110

[7] Thompson, R.S. and Hu, C.R. (1971) Dynamic Structure of Vortices in Superconductors. Physical Review Letters, 27, 1352. http://dx.doi.org/10.1103/PhysRevLett.27.1352 
[8] Knight, J.M. and Kunchur, M.N. (2008) Flux Flow in a Two-Band Superconductor with Delocalized Electric Fields. Physical Review B, 77, Article ID: 024516. http://dx.doi.org/10.1103/PhysRevB.77.024516

[9] Gurevich, A. (2003) Enhancement of the Upper Critical Field by Nonmagnetic Impurities in Dirty Two-Gap Superconductors. Physical Review B, 67, Article ID: 184515. http://dx.doi.org/10.1103/PhysRevB.67.184515

[10] Kunchur, M.N. (1995) Novel Transport Behavior Found in the Dissipative Regime of Superconductors. Modern Physics Letters B, 9, 399. http://dx.doi.org/10.1142/S0217984995000371

[11] Kunchur, M.N. (2004) Current-Induced Pair Breaking in Magnesium Diboride. Journal of Physics: Condensed Matter, 16, R1183. http://dx.doi.org/10.1088/0953-8984/16/39/R01

[12] Pogrebnyakov, A.V., et al. (2004) Properties of $\mathrm{MgB}_{2}$ Thin Films with Carbon Doping. Applied Physics Letters, 85, 2017. http://dx.doi.org/10.1063/1.1782258

[13] Kunchur, M.N., Liang, M. and Gurevich, A. (2012) Thermally Activated Dynamics of Spontaneous Perpendicular Vortices Tuned by Parallel Magnetic Fields in Thin. Physical Review B, 86, Article ID: 024521. http://dx.doi.org/10.1103/PhysRevB.86.024521

[14] Arcos, D.H. and Kunchur, M.N. (2005) Suppressed Flux Motion in Magnesium Diboride Films. Physical Review B, 71, Article ID: 184516. http://dx.doi.org/10.1103/PhysRevB.71.184516 